Barbosa Ramos, Regina.

Profesora Maestra de la Universidad Anhembi Morumbi, Bacharelado en Diseño de Moda, Doctorado en curso en el PPG Design Universidad Anhembi Morumbi.

\title{
Prado, Gilberto.
}

Profesor Doctor del PPG Design Universidad Anhembi Morumbi.

\section{Activación por el Diseño: colaboración y co-creación}

\section{Activation by Design: collaboration and co-creation}

TIPO DE TRABAJO: comunicación virtual.

\section{PALABRAS CLAVE}

Diseño; Activación de discursos; dis-rupción; colaboración; co-creación; activismo.

\section{KEY WORDS}

Design, Discourse activation, disruption, collaboration, co-creation, activism.

\section{RESUMEN}

En el presente artículo, deseamos abordar procesos y procedimientos en Diseño que solicitan del campo activar discursos y proporcionar cambios de comportamiento que afectan la propia práctica del Diseño y la percepción sobre el área. A partir de la adopción y activación de discursos disruptivos, vemos el distanciamiento de la idea de diseño sólo como productor de bienes de consumo, y entendemos que los objetos -y las etapas para producirlos- pueden ser "adoptados" a fin de activar nuevos sentidos que los reubican en el escenario y los hacen promotores de activismo.

\section{ABSTRACT}

In this present article, we desire to approach processes and procedures in Design that demand from this area to activate discourses and provide changes of behavior that affect the very practice of Design and the perception about this area. From the adoption and activation of disruptive discourses, we can perceive the distancing from the idea of design only as a producer of consumer goods, and start to understand that the objects - and the stages to get them produced - can be "adopted" in order to activate new senses that re-locate them in this scene and make them agents and promotes of activism.


Yoko Ono, "Acorn"

Ann Thorpe (2011) define activismo como la toma de acción alineada a las líneas de sostenibilidad, enfatizando metas a largo plazo, el bienestar social y ambiental, a fin de promover cambios a favor de un grupo excluido o descuidado. En la búsqueda de definir Diseñoactivismo, visita una serie de autores y artefactos, delineando parámetros a partir de los cuales entiende que el activismo, además del aspecto disruptivo, tiene siempre como características revelar, desentrañar, o delimitar un problema. 
El enfoque es particularmente interesante al proporcionar la discusión acerca del uso de los medios y mecanismos del Diseño a fin de producir objetos activadores de discursos y promotores de soluciones que subvierten lógicas de mercado.

Así:

\begin{abstract}
... la propuesta del activismo es proveer un desafío más enfocado a los patrones dominantes de poder y subvertirlos en favor de algo mejor. En ese sentido los conceptos de disrupción, encuadrar, desvelar y grupos descuidados se convierten en herramientas para la elaboración de proyectos de diseño activistas. ${ }^{1}$ (Thorpe, 2011, p. 14)
\end{abstract}

A aquellos comprometidos en estos proyectos cabe a menudo la subversión a la manera descrita por De Certeau (2008), en la que se escapa del poder sin dejarlo, y los objetos son entendidos en una instancia dominante en la que la producción encierra significados que difieren de las circunstancias de uso, forjando una "producción secundaria que se esconde en los procesos de su utilización" (De Certeau, 2008, p. 40). El autor menciona que en el que los hechos populares, la construcción de la lengua y las tácticas de supervivencia cotidianas también escapan a las esferas de dominación por medio de una producción-tangible o no- de contenidos inesperados.

Así, análogamente, el diseñador activista asume la responsabilidad por la generación de discursos por medio de lo que produce, en que da a conocer las cuestiones sobre las cuales desea manifestarse, y puede construir espacio de protesta y lugar de activismo por medio del Diseño.

De esta manera, creemos en proyectar a partir del entendimiento del campo como activador de discursos y críticas. La activación, así, pasa a ser el punto de partida para la parametrización de los proyectos a realizar, cuyos productos resultan de diálogos entre áreas, prácticas y personas, en que el Diseño es una actividad proyectual realizada de personas, para personas y con personas, y que tiene como variable imprescindible al usuario, visto como el motivador de esa producción a quien el diseñador va a atender en problemas reales identificados.

Por otro lado, en aquellos procesos que llamamos activados por el diseño, existe la llamada al compromiso, y el entendimiento del diseñador como parte activa de la sociedad que debe acercarse al grupo que pretende atender tanto como pueda, a quien si demanda la construcción de un área de diálogo que permita construir discursos, comunidades y productos que respeten un código de ética de aquel medio.

De esta manera, se constituyen posibilidades de interacción con el usuario por medio de la colaboración. Fletcher y Grose (2011) definen Diseño colaborativo como aquel que se desarrolla a partir de la participación del usuario. Es decir, el proyecto existe, pero el objeto sólo se materializa a partir de la intervención de éste. Sin él el producto es potencia. A partir de él, se hace realidad.

Este tipo de proyecto no se restringe a los bienes materiales, sino también a la promoción de experiencias, como propuesto por la aplicación $\mathrm{N}^{2}$, desarrollado por el músico Jorge Drexler ${ }^{3}$ y el Samsung ${ }^{4}$, diseñado para tablets y smartphones en los que se dan al usuario tres opciones de interacción con composiciones musicales, con un número muy difícil de medir de combinaciones posibles. Cada uno de los $\mathrm{N}$ (figuras) tiene una cantidad de insumos, sujetos a variaciones más o menos complejas y que dan al usuario la sensación de ser, de cierta forma, autor en aquel proyecto.


Figuras: Pantallas de presentación e interacción de los N 1, 2 y 3 disponibles en la aplicación. Imágenes tomadas de: http://www.annacarreras.com/esp/n-album-interactivo-jorge-drexler/

\footnotetext{
1 Traducción de la autora

2 Para mayor información, se puede ver el vídeo de presentación del proyecto $\mathrm{N}$ disponible en

https://www.youtube.com/watch?v=PycgYziA5uM\&list=RDPycgYziA5uM\#t=68 (accedido el 14 de abril de 2017 a las 19:53).

${ }^{3}$ El músico uruguayo, ganador del Oscar por la banda sonora de Diarios de Bicicleta y 14 veces nominado al Grammy.

${ }^{4}$ Samsung es una corporación transnacional surcoreana que actúa en diversas áreas de tecnología de la información, fundada en 1938 y con sede en Seúl, Corea del Sur.
} 
Ahora bien, existe un equipo de diseñadores, programadores, ingenieros entre otros profesionales, además de los músicos, para generar los inputs, pero la canción sólo existe después de esa intervención del usuario. Al término de la experiencia, se ofrecen las opciones de rehacer, grabar y compartir la versión producida por él en las redes sociales.

Ofrecer al usuario la percepción de que la salida de lo que selecciona es virtualmente único es lo que mueve algunas marcas de diseño en el sentido de la colaboración. Como ejemplo, se puede citar la marca de bolsos Guardamundo ${ }^{5}$, con sede en São Paulo, SP. Los modelos de bolsos se ofrecen con un número limitado de materiales y colores a aplicar en las diferentes partes y herrajes (figura).



Figura: Bolsa Modelo Cláudia, tamaño medio en la pantalla inicial de interacción. El usuario puede elegir entre los materiales disponibles para laterales, tirantes, forro, centro y herrajes, las combinaciones que desee. Algunos materiales no generan aumento de precio inicial de la pieza. Imagen tomada de: https://www.guardamundo.com.br/crie-a-sua/22

El número de combinaciones posibles es bastante vasto, lo que confiere al producto finalizado la característica de una muy posible exclusividad. (figuras).

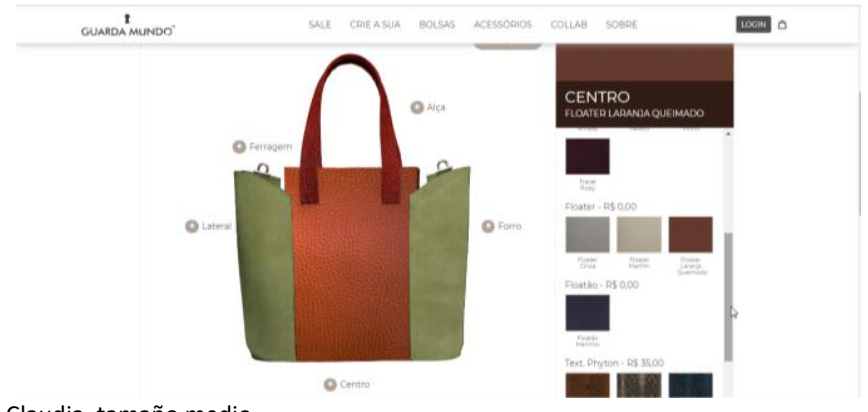

Figura: Simulación de Montaje de Bolsa Claudia, tamaño medio. Disponible: https://www.guardamundo.com.br/crie-a-sua/22

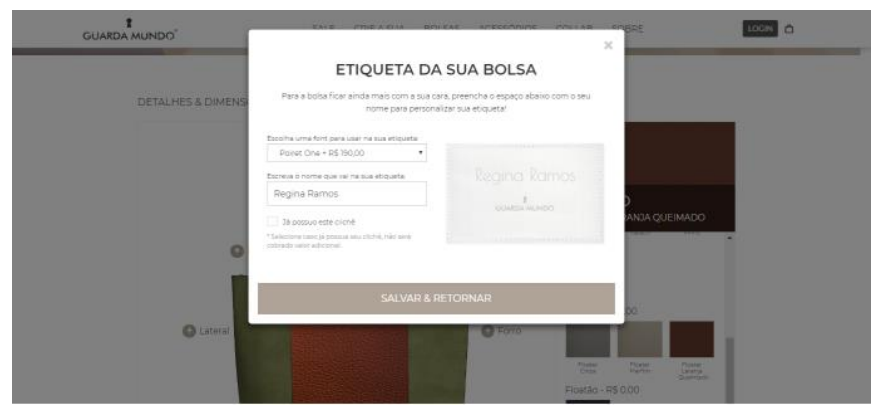

Figura: Simulación de etiqueta personalizada para Bolsa Claudia, tamaño medio. El usuario puede elegir la fuente y escribir el texto. Disponible: https://www.guardamundo.com.br/crie-a-sua/22

Los diseñadores ofrecen el servicio online y producir a demanda, lo que viabiliza su negocio y mantiene su propuesta de valor, sin que los precios se queden exorbitantes.

Los ejemplos están conectados a la relación de los sujetos con la Red y las nuevas tecnologías ya la capacidad de proyectar situaciones que les permitan "llenar" con contenidos 'biográficos' y con los propios 'talentos' el espacio que la Red propone al usuario, para luego trasladarlos a la vida real, espacialmente en su modo de relacionarse y trabajar "(Morace, 2009, p. 11).

${ }^{5}$ Disponível em http://www.guardamundo.com.br/ (acesso em 3 de Janeiro de 2017 às 13:23). 
De esta manera, se dibuja al usuario como un protagonista en un proceso colaborativo, pero no necesariamente en un proyecto colectivo. Concepción y creación existen antes del sujeto usuario y, aunque su existencia ha sido tenida en cuenta y el producto sólo pasa a existir de él, no siempre el usuario está comprometido en todas las dimensiones de los discursos adoptados por los autores. Y es esa diferencia de actuación en la concepción del proyecto que merece destaque, pues es ahí donde se dibuja el escenario para la activación de diálogo a través del Diseño, en que es necesario que todos los sujetos sean conscientes de su participación y contribuyan no sólo con sus habilidades prácticas, sino con la comprensión del valor de su participación.

\section{Desarrollo colectivo de proyectos de diseño}

Contemporáneamente se observa la proliferación de grupos que desarrollan proyectos que involucran intercambios significativos a fin de concebir procesos y productos en que se permite a los sujetos que, asociados, tragan a la superficie sus repertorios, recursos, perspectivas y percepciones al respecto de lo que se entiende por problemas en Diseño y la búsqueda de soluciones para los mismos.

Tales actividades pueden ser vistas tanto como formadoras de "comunidad como un movimiento con valores atractivos, del cual las personas desean formar parte"6 (Gauntlett, 2018, p. 75) "una reacción contra una enorme cantidad de cosas, incluyendo nuestra cultura hiper-rápida, creciente dependencia de las tecnologías digitales, y la proliferación de la cultura de consumo"7 (Gschwandtner, 2008, apud Gauntlett, 2018, p. 75).

Los colectivos se organizan por voluntad propia y en ejercicio libre de reconocimiento, y no en coexistencia forzada. En "Comunidad", Zygmunt Bauman investiga las relaciones comunales, sociales, y la construcción de nación e identidad nacional, en abierta crítica a los discursos neoliberales acerca del multiculturalismo y la pluralidad, que, a su ver, apenas disfrazan la dominación de los valores de los valores comunidades "más fuertes" sobre las "más débiles". Así, cabría a las comunidades más débil ser asimiladas - y, con ello, tener aquello que las diferencia de la "nación" aniquilado -o perecer - y, consecuentemente, tener el diferente aniquilado. "Cualquiera que fuera la solución adoptada," ninguna de ellas dejaba espacio para la supervivencia de la comunidad "(Bauman, 2003, p. 85), porque la percepción de" comunidad "vigente es aquella que teme lo que es" diferente "y se dispone a la" "Dialogar" sólo con lo que se identifica, sin provocar tensiones.

Así, a fin de consolidar una idea de "comunidad" fundamentada en los discursos hegemónicos, el "diferente", el "otro", irreconocido y irrespetuoso, debería ser empujado hacia lejos, confinado en guetos, en que se "disuelve la solidaridad y destruye la confianza mutua antes de que éstas hayan tenido tiempo de crear raíces ", en que se vive la" imposibilidad de comunidad "(Bauman, 2003, pp. 110-11).

Los colectivos, sin embargo, se constituyen, desde la perspectiva baumaniana, como comunidades, pues se lanzan en bases que son evidenciadoras de las diferencias, buscan mostrar sus valores (éticos, estéticos, políticos, poéticos que sean) y traerlos a y se ocupan de la consolidación de las áreas de diálogo, incluso cuando pueden causar más fricciones que acomodaciones (incluso entre los participantes). Tal perspectiva encuentra la lectura de Salles, para quien "el propio sujeto tiene la forma de una comunidad; la multiplicidad de interacciones no implica absoluta supresión del sujeto y el locus de la creatividad no es la imaginación de un individuo "(Salles, 2014, p 152), en que la autoría se establece dinámicamente en las relaciones entre los participantes del colectivo.

Esas comunidades -los colectivos- formuladas en el entorno del hacer, organizadas por la búsqueda de formar vínculos significativos, nos llevan al modelo de los sujetos peninsulares, acuñado por Amós Oz, para quienes:

... ningún hombre y ninguna mujer es una isla, pero cada uno de nosotros es una península, mitad ligada al continente, mitad hacia el mar (...). Creo que deberíamos tener permiso para seguir siendo penínsulas. $(O z, 2016$, p. 82)

Proponer que cada uno de los involucrados permanezca península es solicitar que estén conectados, pero que sea preservado aquel conjunto de recursos que los individualiza. Creemos que esa relación que no amalgama a los sujetos proporciona el desarrollo de proyectos en los que la propuesta de diálogo es principio inatacable. Un "encuentro de penínsulas", en el que se está "cerca el uno del otro, a veces muy cerca, pero sin apagarse. Sin asimilarse. Sin anular la individualidad "(Oz, 2017, p. 47).

Para el Diseño, desde siempre una área interdisciplinaria, parece coherente que los involucrados en los proyectos se vean a sí mismos como un grupo en que la multiplicidad de las miradas, que es capaz de construir un área de diálogo que comporte los diferentes repertorios, autorizando así la ocurrencia de múltiples posibilidades de abordaje de las cuestiones planteadas para los proyectos realizados, en que "la comparación entre socios es entendido como sin importancia"8 (Gauntlett, 2018, p. 77).

\footnotetext{
6 Tradución de la autora.

7 Tradución de la autora.

8 Tradución de la autora.
} 
Entendemos entonces que los colectivos operan a partir del reconocimiento de identidades y alteridades, en que los encuentros entre las partes son creadores de un espacio de negociaciones, convergencias y tensiones. También está acordado que se organizan en relaciones heterárquicas, en que los operadores son deseablemente autónomos y buscan actuar en consenso.

En este contexto, se construyen relaciones dinámicas y oriundas de la percepción de un panorama crítico, en el que se hace necesario romper con posiciones cristalizadas acerca de las ideas de producción, con la materialidad y los modos de existir:

... cuando las viejas historias de filiación (comunitaria) ya no suenan verdaderas al grupo, crece la demanda por "historias de identidad" en que "decimos a nosotros mismos de dónde venimos, quiénes somos y hacia dónde vamos"; tales historias son urgentemente necesarias para restaurar la seguridad, construir la confianza y hacer "posible la interacción significativa con otros." (Bauman, 2003, p. 90)

Diferentemente de los "estilos de vida" en que se popularizaron las libertades de parecer con lo que bien se entiende, y pertenecer, aunque momentáneamente, a cualesquiera grupos que se eligen, mucho más por gusto que por creencia (Lipovetsky, 2009), se encuentran los modos de existir, en que los individuos buscan formular una construcción ética de existencia, fundamentada en elecciones conscientes y que mapean los valores y relaciones de los individuos con los demás y con los ambientes en que se insertan.

Así, el diseño contemporáneo de colectivo adopta prácticas que corren para producir espacios en que el diálogo permite visibilizar las cuestiones del grupo, promover relaciones inter y transdisciplinares posibilitadas por la existencia de las particularidades de los sujetos involucrados, capaces de "introducir y facilitar el flujo de" (Hickel, 2015, p. 80) y soluciones que pueden ser "ideas mejores", disruptivas ciertamente desestabilizadoras - en mayor o menor medida.

Los colectivos de diseño contemporáneos tienen como propuesta, en gran parte, producir discursos, apoyados o no por objetos. En el caso de los artistas y / o los diseñadores, tanto si producen obras materiales, inmateriales, perennes, efímeras, interactivas o no, en colectivo la heterarquía de las relaciones es un factor primordial, pues se espera que los diferentes operadores contribuyan a la completitud de la obra con aquello de que disponen, "posibilitando la autonomía de cada participante para encontrar su propio modo de estar en el proceso" (Gois, Bonfim y Brito, in Cirillo et al., 2015, p. 179). De esta manera, lo que se evidencia es la autonomía y la autogestión, la naturaleza dinámica de las inferencias y las posibilidades de rediseño de proyecto en curso, a partir de las contribuciones y competencias identificadas de los participantes, para quienes los procesos "auxilian a las personas a aprender y crear vínculos "9 (Gauntlett, 2018, p. 78).

Además, los colectivos contemporáneos, de acuerdo con Spampinato, hacen de sus trabajos una invitación para conversar con el público, oportunizan nuevos diálogos, pues como productores y público, experimentan el proceso de hacer, más atentos a los detalles ya las decisiones que fundamentan las elecciones cotidianas acerca de los objetos construidos y la experiencia de utilizarlos (Gauntlett, 2018, p. 71).

Sin embargo, organizarse en colectivo, supone crear la oportunidad para desarrollar conexiones sociales y crecimiento personal (Gauntlett, 2018), a partir del reconocimiento de las diferencias y de las consecuentes tensiones derivadas del no supresión del individuo, del "reconocimiento del derecho igual para todos los seres humanos de ser diferentes unos de otros y de cada individuo aislado se equipar a un mundo entero, cuya existencia merece respeto "(Oz, 2017, p. 59).

Así, se reescriben las narrativas y actividades comunales y se hace evidente que, al optar por trabajar colectivamente, el grupo asume un modus operandi, y también hace de él el mensaje, o al menos construye parte de ella a partir de su conformación, en que los operadores de un colectivo no son mayores que las causas que defienden, que las cuestiones que plantean, que las propuestas que hacen.

Acerca de las tensiones, estamos de acuerdo con Oz (2017, pp. 59-60), cuando dice que "la controversia no es una situación de debilidad preocupante, sino un clima positivo para el florecimiento de una vida creativa" que converge con la " "Noción de contrapunto y también con la de polifonía humana, en la cual la comunidad es un coro de muchas y diferentes voces, una orquesta con instrumentos variados, todos regidos en un sistema de reglas consensuadas" (idem, ibidem, p. 69).

En resumen, los colectivos de Diseño se organizan, a la guisa de comunidades, por voluntad propia, en torno al hacer. En la mayoría de los casos, los cambios significativos realizados en situaciones heterarquicas (el poder descentralizado de responsabilidades compartidas), en que lo importante es la contribución hecha durante el proyecto.

Los participantes de los colectivos traen a la mesa de trabajo sus habilidades y los deseos de aprender y oportunamente enseñar, en un movimiento que privilegia la intercambio y construcción de conocimiento. Los coordinadores de proyecto, así, funcionan como proponentes y facilitadores de la formulación de las áreas de diálogos que seguramente serán establecidas entre aquellos que se involucran en situaciones de proyección en colectivo.

9 Tradución de la autora. 
Las áreas de diálogo así constituidas delinean - pero no limitan - el terreno necesario para la experimentación y la innovación, definida por Coelho (2008, p. 103), como la introducción de novedades en lo existente, pero que, de alguna manera, la forma como ese existente previo se presenta, a punto de parecer nuevo, como una invención. ${ }^{10}$

Para el Diseño, implica acercarse a un proceso de aceptación de que los proyectos pueden ser más que realizar lo que fue previamente concebido. La realización de un proyecto de diseño pasa a ser un camino de descubrimiento en el que las rutas siempre se vuelven a enriquecerse, en que nuevas y mejores ideas pueden ser formuladas durante el proceso de hacer.

\section{CONSIDERACIONES FINALES}

Nuestra percepción de Diseño Dialógico, permea relaciones, polifónicas, heterárquicas y ecuánimes. Para nosotros, el pensamiento de complementariedad causa una incomodidad pues vemos el Diseño Dialógico, fundamentado sobre nuestra lectura entrelazada de los modelos que elegimos, leída, mayoritariamente, con las presencias, semejanzas, reconocimientos (de las diferencias, inclusive) y deseos comunes y menos con las ausencias que la complementariedad presupone, y permite al diseñador colocarse en un contexto en el que puede experimentar diferentes y convergentes modos de existir.

Elegimos la investigación de procedimientos colectivos en Diseño activando diálogos que abordan pautas concernientes a grupos externos al colectivo, e incluso verse estimulado a los grupos repensar sus acciones en función de las cuestiones presentadas por esos agentes externos.

Así, si "acoge por tanto la miscelánea colaborativa, la construcción combinada de las peculiaridades y talentos individuales, una celebración de la imperfección, de la imaginación, y del" hacer lo que usted puede "11 (Gauntlett, 2018, p. 38) y delinea 12 el área de diálogo en la que es posible proyectar juntos un futuro posible para el diseño y para los diseñadores.

\section{REFERENCIAS BIBLIOGRÁFICAS}

Bauman, Z. (2003). Comunidade: a busca por segurança no mundo atual. Rio de Janeiro: Ed. Zahar.

De Certeau, M. (2008). A Invenção do Cotidiano: artes de fazer. Rio de Janeiro: Ed. Vozes.

Cirillo, J., Kinceler, J., Oliveira, L. (Eds.) (20015). Outro Ponto de Vista: práticas colaborativas na arte contemporânea. Vitória: PROEX/UFES.

Coelho, L. (Ed.) (2008). Conceitos-Chave em Design. Rio de Janeiro: Ed. PUC-Rio. Novas Ideias.

Fletcher, K.; Grose, L. (Eds.) (2011). Moda \& Sustentabilidade, Design Para Mudança. São Paulo: Editora Senac.

Ganem, M. (2016). Design Dialógico: gestão criativa, inovação e tradição. São Paulo: Estação das Letras e Cores.

Gauntlett, D. (2018). Making Is Connecting: the social power of creativity, from craft and knitting to digital everything. Medford: Polity.

Hickel, D. (2015, Julio). O Design Como a Expressão de Fazer as Coisas Juntos: um entendimento ecológico. Projética, Londrina, 6 (1), 77-96.

Korn, P. (2016). Why We Make Things and Why it Matters: the education of a craftsman. Boston: David R. Godine Publisher.

Lipovetsky, G. (2009). O Império do Efêmero: a moda e seu destino nas sociedades modernas. São Paulo: Companhia das Letras.

Morace, F. (2009). Consumo Autoral: as gerações como empresas criativas. São Paulo: Estação das Letras e Cores Editora.

\footnotetext{
10 Optamos por presentar brevemente y conceptualmente las ideas de invención e innovación. Coelho entiende que la innovación es un proceso de descristalización de los sentidos de un signo, "permitiendo nuevas asociaciones entre sus elementos" (COELHO, 2008, p. 102), mientras que la invención se refiere al ineditismo, a la primera aparición de aquello. (Nota de la autora)

11 Tradución autora.

12 Y no se delimita.
} 
Barbosa Ramos, Regina; Prado, Gilberto

Activación por el Diseño: colaboración y co-creación

IV Congreso INTERNACIONAL DE INVESTIGACIÓN EN ARTES VISUALES ANIAV 2019 IMAGEN [N] VISIBLE]

http://dx.doi.org/10.4995/ANIAV.2019.8996

Oz, A. (2016). Como Curar Um Fanático: Israel e Palestina: entre o certo e o certo. São Paulo: Companhia das Letras.

Oz, A. (2017). Mais de Uma Luz: fanatismo, fé e convivência no século XXI. São Paulo: Companhia das Letras.

Salles, C. (2014). Redes da Criação: construção da obra de arte. São Paulo: Editora Horizonte.

Spampinato, F. (2015). Come Together, the rise of cooperative art and design. Nova lorque: Princeton Architectural Press.

Thorpe, A. (2011). Defining Design As Activism. Artigo submetido ao Journal of Architectural Education. 